\title{
PROPERTIES OF THE CORE-MANTLE BOUNDARY
}

\author{
Bradford H. Hager \\ Seismological Laboratory \\ California Institute of Technology \\ Pasadena, CA 91125 USA
}

The core-mantle boundary (CMB), separating the molten metallic core from the overlying solid silicate mantle, marks the largest discontinuity in mechanical properties within the Earth. The $\sim 200 \mathrm{~km}$ thick region just above the CMB, named $D^{\prime \prime}$ by Bullen (1950), is characterized by an anomalous gradient in seismic velocity versus depth. $D^{\prime \prime}$ was originally interpreted as a region with a strong compositional gradient due to the accumulation of dense material at the base of the mantle. Subsequently, the anomalous gradient was interpreted as the result of a strong temperature gradient in a hot thermal boundary layer at the base of the mantle, an interpretation motivated by the requiremnet that heat involved in generating the geodynamo must be transported out of the core and through the mantle by convection.

Determining the nature of $D^{\prime \prime}$ is important to understanding the geochemical evolution of Earth, in addition to understanding mantle and core dyanmics. If $D^{\prime \prime}$ is compositionally distinct from the mantle, it represents a reservoir of material not yet considered in modeling the composition of the mantle. Since direct sampling is impossible, we must resort to indirect methods to determine its properties.

Mapping the topography fo the CMB is one way of constraining the nature of $D^{\prime \prime}$. Due to the high temperature near the CMB, mantle materials respond to stresses by creeping flow; any topographic relief on the CMB must be dynamically maintained by convection in the overlying mantle. If $D^{\prime \prime}$ were a separately convecting layer, this topography would be much smaller than if $D^{\prime \prime}$ were the thermal boundary layer of the (entire) convecting lower mantle. CMB topography can be probed in a number of ways. Nutational coupling of the core and mantle depends upon CMB ellipticity. Torques caused by the interaction of fluid flow in the core and CMB topography would cause changes in the length of day. The predicted dynamic topography of the CMB caused by lower mantle density contrasts inferred from seismic tomography can be calculated from fluid mechanical models. The variations in travel times of seismic waves that reflect off or are transmitted through the CMB can be mapped into models of CMB topography.

The results from a number of recent models of CMB topography coming from various branches of geophysics are not in agreement. Nutation and length of day studies indicate CMB topography of order hundreds of meters, while seismic models have topography of over $10 \mathrm{~km}$. Dynamic models that include $D^{\prime \prime}$ as a chemically distinct layer are in accord with the former estimates. 